\title{
The gravitational energy-momentum tensor and the gravitational pressure
}

\author{
J. W. Maluf* \\ Instituto de Física, \\ Universidade de Brasília \\ C. P. 04385 \\ 70.919-970 Brasília DF, Brazil
}

\begin{abstract}
In the framework of the teleparallel equivalent of general relativity it is possible to establish the energy-momentum tensor of the gravitational field. This tensor has the following essential properties: (1) it is identified directly in Einstein's field equations; (2) it is conserved and traceless; (3) it yields expressions for the energy and momentum of the gravitational field; (4) is is free of second (and highest) derivatives of the field variables; (5) the gravitational and matter energy-momentum tensors take place in the field equations on the same footing; (6) it is unique. However it is not symmetric. We show that the spatial components of this tensor yield a consistent definition of the gravitational pressure.
\end{abstract}

PACS numbers: 04.20.Cv, 04.20.Fy

(*) e-mail: wadih@fis.unb.br 


\section{Introduction}

A comprehensive understanding of Einstein's general relativity requires not only the knowledge of the structure of the field equations, the solutions and physical consequences, but also the understanding of properties such as the energy, momentum and angular momentum of the gravitational field. These properties have been addressed in the teleparallel equivalent of general relativity (TEGR) in the past few years. The TEGR $[1,2,3,4,5]$ is just a reformulation of Einstein's general relativity in terms of tetrad fields and the torsion tensor. It is not a new theory for the gravitational field, because the field equations for the tetrad field are precisely equivalent to Einstein's equations. The TEGR is just an alternative geometrical formulation of general relativity, and therefore it provides an alternative insight into the theory. Investigations on pseudo-tensors indicated that a possible expression for the gravitational energy density would be given in terms of second order derivatives of the metric tensor. It is known that such expression, covariant under arbitrary changes of coordinates, does not exist. For this reason investigations on quasi-local gravitational energy - the energy contained within a closed spacelike two-surface - have been carried out (a recent review on the subject is given in Ref. [6]). However a covariant expression that is linear in the derivatives of the torsion tensor can be constructed, and in fact a definition for the gravitational energy-momentum has been presented $[7,8]$ and thoroughly investigated in the framework of the TEGR. We recall that the torsion tensor cannot be made to vanish at a point in space-time by means of a coordinate transformation. Therefore criticisms based on the Principle of Equivalence, which rest on the reduction of the metric tensor to the Minkowski metric tensor at a point in space-time by means of a coordinate transformation, do not apply to the above mentioned definition. It has been argued [9] that the fact that the first derivatives of the metric tensor can be made to vanish not only along the world line of a freely falling observer, but along any world line, and independently of whether the metric tensor obeys any field equations, is just a feature of differential geometry.

The quasi-local expressions for the gravitational energy-momentum are obtained by means of two general procedures [6]: the Lagrangian approach (the quasi-local quantities are integrals of superpotentials derived from the Lagrangian via a Noether-type analysis) or the Hamiltonian approach (the quasi-local quantities are the values of the Hamiltonian on the constraint 
surface, in the phase space, as in the Regge and Teiltelboim [10] analysis). However in both approaches the resulting expressions are not uniquely determined because the action can be modified by adding an (almost freely chosen) boundary term to it (see section 3.3.3 of Ref. [6]). In contrast, in the framework of the TEGR the gravitational energy-momentum $P^{a}$ is identified directly in the field equations. The Hamiltonian analysis of the TEGR was crucial to identifying $P^{a}$. It was first observed [4] that the Hamiltonian constraint contains a scalar density in the form of a total divergence. The emergence of such quantity is possible in theories constructed out of the torsion tensor. This scalar density is identified as the gravitational energy density because integration of the latter leads to satisfactory values of the grativational energy for several distinct configurations of the gravitational field. The integral form of the Hamiltonian constraint equation is then interpreted as an equation for the gravitational energy of the type $H-E=0$. In this formulation the time gauge condition was imposed from the outset. In the more general Hamiltonian formulation [11] the Hamiltonian and vector constraints also contain a total divergence, the integral form of which leads to the definition of $P^{a}$.

The Lagrangian formulation of the TEGR is much simpler than its Hamiltonian formulation. The identification of $P^{a}$ in the Lagrangian field equations leads, after very simple algebraic manipulations, to a continuity equation for the gravitational energy-momentum, to conservation laws for $P^{a}=(E, \mathbf{P})$ and consequently to a definition of the gravitational energy-momentum flux $[12,13]$. It is clear that in the framework of the TEGR several issues regarding the gravitational energy-momentum can be addressed and investigated.

In this article we obtain the energy-momentum tensor of the gravitational field directly from the Lagrangian field equations. This tensor yields the energy-momentum, the flux of energy-momentum and the stresses of the gravitational field. The immediate consequence of this analysis is the definition of gravitational pressure. We have applied this definition to the simple case of the Schwarzschild space-time. We hope that the present definition may prove to be useful in analises of the thermodynamics of the gravitational field.

Notation: space-time indices $\mu, \nu, \ldots$ and $\mathrm{SO}(3,1)$ indices $a, b, \ldots$ run from 0 to 3 . Time and space indices are indicated according to $\mu=0, i, \quad a=(0),(i)$. The tetrad field and the $\mathrm{SO}(3,1)$ connection are denoted by $e^{a}{ }_{\mu}$ and $\omega_{\mu a b}$, repectively. The flat, Minkowski space-time metric tensor raises and lowers 
tetrad indices and is fixed by $\eta_{a b}=e_{a \mu} e_{b \nu} g^{\mu \nu}=(-+++)$. The determinant of the tetrad field is represented by $e=\operatorname{det}\left(e^{a}{ }_{\mu}\right)$.

\section{The teleparallel equivalent of general rela- tivity}

Let us consider a four-dimensional pseudo-riemannian manifold endowed with a set of tetrad fields $e^{a}{ }_{\mu}$ and a $\mathrm{SO}(3,1)$ (spin) connection $\omega_{\mu a b}$. These quantities define the metric tensor $g_{\mu \nu}=e^{a}{ }_{\mu} e_{a \nu}$, the torsion tensor,

$$
T_{\mu \nu}^{a}(e, \omega)=\partial_{\mu} e^{a}{ }_{\nu}-\partial_{\nu} e^{a}{ }_{\mu}+\omega_{\mu}{ }_{b}{ }_{b} e^{b}{ }_{\nu}-\omega_{\nu}{ }_{b}{ }_{b} e^{b}{ }_{\mu},
$$

the curvature tensor,

$$
R_{b \mu \nu}^{a}(\omega)=\partial_{\mu} \omega_{\nu}{ }_{b}{ }_{b}-\partial_{\nu} \omega_{\mu}{ }^{a}{ }_{b}+\omega_{\mu}{ }^{a}{ }_{c} \omega_{\nu}{ }^{c}{ }_{b}-\omega_{\nu}{ }^{a}{ }_{c} \omega_{\mu}{ }^{c}{ }_{b},
$$

and the scalar curvature,

$$
R(e, \omega)=e^{a \mu} e^{b \nu} R_{a b \mu \nu}(\omega) .
$$

The equation that defines the torsion tensor can be solved for $\omega_{\mu a b}$. After some manipulations it is possible to obtain the identity,

$$
\omega_{\mu a b}={ }^{0} \omega_{\mu a b}(e)+K_{\mu a b} .
$$

where ${ }^{0} \omega_{\mu a b}(e)$ is the metric compatible Levi-Civita connection, and

$$
K_{\mu a b}=\frac{1}{2} e_{a}^{\lambda} e_{b}^{\nu}\left(T_{\lambda \mu \nu}+T_{\nu \lambda \mu}+T_{\mu \lambda \nu}\right),
$$

is the contorsion tensor.

Substitution of the identity (4) into Eq. (3) yields an identity that relates the scalar curvature given by Eq. (3) with the scalar curvature $R\left({ }^{0} \omega\right) \equiv R(e)$ constructed out the tetrad field $e^{a}{ }_{\mu}$ only,

$$
\begin{aligned}
e R(e, \omega) & =e R(e) \\
& +e\left(\frac{1}{4} T^{a b c} T_{a b c}+\frac{1}{2} T^{a b c} T_{b a c}-T^{a} T_{a}\right)-2 \partial_{\mu}\left(e T^{\mu}\right),
\end{aligned}
$$


where $T_{a}=T_{b a}^{b}=T_{\mu a}^{\mu}$ and $T_{a b c}=e_{b}{ }^{\mu} e_{c}{ }^{\nu} T_{a \mu \nu}$. As usual, the tetrad field converts space-time into $\mathrm{SO}(3,1)$ indices and vice-versa. The tensor $\Sigma^{a b c}$ defined by

$$
\Sigma^{a b c}=\frac{1}{4}\left(T^{a b c}+T^{b a c}-T^{c a b}\right)+\frac{1}{2}\left(\eta^{a c} T^{b}-\eta^{a b} T^{c}\right)
$$

yields

$$
\Sigma^{a b c} T_{a b c}=\frac{1}{4} T^{a b c} T_{a b c}+\frac{1}{2} T^{a b c} T_{b a c}-T^{a} T_{a},
$$

and thus we have

$$
e R(e, \omega)=e R(e)+e \Sigma^{a b c} T_{a b c}-2 \partial_{\mu}\left(e T^{\mu}\right) .
$$

Therefore the vanishing of the curvature tensor $R^{a}{ }_{b \mu \nu}(\omega)$, and consequently of the scalar curvature given by Eq. (3), implies the equivalence of the scalar curvature density $e R(e)$, which defines the Lagrangian density for Einstein's general relativity, with the quadratic combination of the torsion tensor.

We establish the Lagrangian density $L^{\prime}(e, \omega, \lambda)$ for the TEGR with local $\mathrm{SO}(3,1)$ symmetry according to

$$
L^{\prime}(e, \omega, \lambda)=-k e \Sigma^{a b c} T_{a b c}+\lambda^{a b \mu \nu} R_{a b \mu \nu}(\omega)-L_{M},
$$

where $k=1 /(16 \pi G)$ and $L_{M}$ is the Lagrangian density for matter fields. $\left\{\lambda^{a b \mu \nu}\right\}$ are Lagrange multipliers that ensure the vanishing of the curvature tensor $R_{a b \mu \nu}(\omega)$. For asymptotically flat space-times the variation of the scalar $\Sigma^{a b c} T_{a b c}$ is well defined. All surface terms that arise in the integration by parts vanish at spatial infinity by requiring the usual asymptotic conditions on $e_{a \mu}$, and therefore there is no need of addition of surface terms to $L^{\prime}$. Arbitrary variations of $L^{\prime}$ with respect to $e^{a \mu}, \omega_{\mu a b}$ and $\lambda^{a b \mu \nu}$ yield, respectively [4],

$$
\begin{gathered}
e_{a \lambda} e_{b \mu} D_{\nu}\left(e \Sigma^{b \lambda \nu}\right)-e\left(\Sigma^{b \nu}{ }_{a} T_{b \nu \mu}-\frac{1}{4} e_{a \mu} T_{b c d} \Sigma^{b c d}\right)=\frac{1}{4 k} e T_{a \mu}, \\
\Sigma^{a \mu b}-\Sigma^{b \mu a}-\frac{1}{e} D_{\nu}\left(e \lambda^{a b \mu \nu}\right)=-\frac{1}{2} S^{\mu a b}
\end{gathered}
$$




$$
R_{a b \mu \nu}(\omega)=0 .
$$

In Eqs. (11), (12) and (13) we have the following definitions,

$$
\begin{aligned}
D_{\nu}\left(e \Sigma^{b \lambda \nu}\right) & =\partial\left(e \Sigma^{b \lambda \nu}\right)+e \omega_{\nu}^{b}{ }_{c}{ }^{\Sigma^{c \lambda \nu}} \\
D_{\nu}\left(e \lambda^{a b \mu \nu}\right) & =\partial_{\nu}\left(e \lambda^{a b \mu \nu}\right)+e\left(\omega_{\nu}{ }^{b}{ }_{c} \lambda^{a c \mu \nu}+\omega_{\nu}{ }^{b}{ }_{c} \lambda^{c b \mu \nu}\right) \\
\frac{\delta L_{M}}{\delta e^{a \mu}} & =e T_{a \mu} \\
\frac{\delta L_{M}}{\delta \omega_{\mu a b}} & =e S^{\mu a b}
\end{aligned}
$$

The Lagrangian density $L^{\prime}$ as well as the field equations (11), (12) and (13) are invariant under $\mathrm{SO}(3,1)$ and general coordinate transformations.

In order to verify the equivalence of the field equations (11) with Einstein's equations we substitute $\omega_{\mu a b}$ given by Eq. (4) into $0=R_{a \mu}(e, \omega)-\frac{1}{2} e_{a \mu} R(e, \omega)$ (recall that $R_{a b \mu \nu}(\omega)=0$ ). After long but otherwise simple calculations we find

$$
e_{a \lambda} e_{b \mu} D_{\nu}\left(e \Sigma^{b \lambda \nu}\right)-e\left(\Sigma^{b \nu}{ }_{a} T_{b \nu \mu}-\frac{1}{4} e_{a \mu} T_{b c d} \Sigma^{b c d}\right)=e\left[R_{a \mu}(e)-\frac{1}{2} e_{a \mu} R(e)\right]
$$

The expression that appears on the left hand side of the expression above is precisely the same one on the left hand side of Eq. (11). Therefore this feature establishes the equivalence of the TEGR with Einstein's general relativity.

Let us make two remarks regarding the above Lagrangian formulation. First, we note that the right hand side of Eq. (16) does not depend on $\omega_{\mu a b}$, whereas the left hand side does depend on it (this remark was first pointed out in Ref. [4]). Therefore the $\mathrm{SO}(3,1)$ connection $\omega_{\mu a b}$ plays no role in the dynamics of the tetrad field, which ultimately establishes the space-time geometry. Second, we observe that Eq. (12) represents 24 equations. These equations are insufficient to determine the 36 components of the Lagrange multiplier $\lambda^{a b \mu \nu}$. Some of the latter quantities remain undetermined in the theory. From a different perspective, it has been pointed out in Ref. [5] that the Lagrange multipliers may be redefined by means of a symmetry operation, which renders an ambiguity in the determination of the multipliers. 
Of course this is an unsatisfactory feature of the theory. The theory defined without the connection $\omega_{\mu a b}$ is much simpler and free of this undetermination, and leads to the correct dynamics for $e_{a \mu}$. The presence of $\omega_{\mu a b}$ is not mandatory for the mathematical consistency of the theory. Moreover, it has been shown that the Hamiltonian formulation of the theory constructed out of $e_{a \mu}$ only is consistent [11]. Therefore we will not take into account the Lagrangian density $L^{\prime}$, and consider instead the TEGR determined out of $e_{a \mu}$ only. By dropping out $\omega_{\mu a b}$ the theory is no longer invariant under local $\mathrm{SO}(3,1)$ transformations, but rather invariant under global $\mathrm{SO}(3,1)$ transformations. However, the loss of this local symmetry does not imply any restriction on the class of possible frames. Every tetrad field $e_{a \mu}$ that is a solution of the theory with local $\mathrm{SO}(3,1)$ symmetry is also a solution of the theory with global SO $(3,1)$ symmetry. The global $\mathrm{SO}(3,1)$ symmetry implies a rigid geometric structure in space-time that is closer in spirit to the teleparallel geometry, as it allows the definition of distant parallelism. Finally, we will argue later on that the global $\mathrm{SO}(3,1)$ symmetry is an essential feaure of the gravitational energy-momentum, in view of the Principle of Equivalence.

By making $\omega_{\mu a b}=0$ the torsion tensor is simplified to

$$
T_{\mu \nu}^{a}(e)=\partial_{\mu} e^{a}{ }_{\nu}-\partial_{\nu} e^{a}{ }_{\mu},
$$

and the identity (6) is rewritten as

$$
e R(e)=-\left(\frac{1}{4} T^{a b c} T_{a b c}+\frac{1}{2} T^{a b c} T_{b a c}-T^{a} T_{a}\right)+2 \partial_{\mu}\left(e T^{\mu}\right) .
$$

Considering the same definition (7) for the tensor $\Sigma^{a b c}$, we define the Lagrangian density $L(e)$,

$$
L(e)=-k e \Sigma^{a b c} T_{a b c}-L_{M},
$$

constructed out of $e_{a \mu}$ and matter fields only. The field equations derived from arbitrary variations of $L(e)$ with respect to $e_{a \mu}$ are given by

$$
e_{a \lambda} e_{b \mu} \partial_{\nu}\left(e \Sigma^{b \lambda \nu}\right)-e\left(\sum^{b \nu}{ }_{a} T_{b \nu \mu}-\frac{1}{4} e_{a \mu} T_{b c d} \Sigma^{b c d}\right)=\frac{1}{4 k} e T_{a \mu},
$$

where $T_{a \mu}$ is defined by Eq. (14) and $T_{a \mu \nu}$ by Eq. (17). The theory defined by Eq. (19) is equivalent to Einstein's general relativity because it can be shown that the left hand side of the equation above can be rewritten 
as $\frac{1}{2} e\left[R_{a \mu}(e)-\frac{1}{2} e_{a \mu} R(e)\right]$. In the following sections of this article we will consider the theory determined by Eqs. (19) and (20).

\section{The gravitational energy-momentum ten- sor}

The difficulty in arriving at a consistent definition for the gravitational energy-momentum tensor led to investigations on pseudo-tensors. However it has become clear that this difficulty lies in the traditional description of the gravitational field, not in the nature of gravity as such. This point of view is considered, for instance, in the interesting analysis developed in Ref. [9], which attempts to define the gravitational energy-momentum by means of the field-theoretical formulation of general relativity. However, already in Ref. [7] it was antecipated that the metrical description of gravity is not suitable for such purpose. The geometrical formulation of the TEGR has proven to be more adequate for addressing the above mentioned difficulty.

In the Hamiltonian formulation of the TEGR [11] in empty space-time the Hamiltonian and vector constraints, $H_{0}$ and $H_{i}$, respectively, can be arranged in order to determine the constraint $C^{a}, C^{a}=e^{a 0} H_{0}+e^{a i} H_{i}+\cdots$, which in turn can be written as $C^{a}=-\partial_{i} \Pi^{a i}-H^{a}$, where $\Pi^{a i}$ is the momentum canonically conjugated to $e_{a i}$, and $H^{a}$ is defined as the remaining part of $C^{a}$. The integral form of the constraint equations $C^{a}=0$ is interpreted as an equation that defines the vacuum gravitational energy-momentum $P^{a}$ [8],

$$
P^{a}=-\int_{V} d^{3} x \partial_{j} \Pi^{a j}
$$

where $\Pi^{a j}=-4 k e \sum^{a 0 j}$. This definition has been applied quite satisfactorily to several gravitational field configurations.

Returning to the Lagrangian formulation, by properly identifying $\Pi^{a i}$ in the field equations (20), after some simple algebraic manipulations it is possible to arrive at a continuity equation for the gravitational energy-momentum $[12,13]$,

$$
\frac{d}{d t}\left[-\int_{V} d^{3} x \partial_{j} \Pi^{a j}\right]=-\Phi_{g}^{a}-\Phi_{m}^{a}
$$


where $V$ is an arbitrary volume in the three-dimensional space,

$$
\Phi_{g}^{a}=k \oint_{S} d S_{j}\left[e e^{a \mu}\left(4 \Sigma^{b c j} T_{b c \mu}-\delta_{\mu}^{j} \Sigma^{b c d} T_{b c d}\right)\right],
$$

is the $a$ component of the gravitational energy-momentum flux, and

$$
\Phi_{m}^{a}=\oint_{S} d S_{j}\left(e e_{\mu}^{a} T^{j \mu}\right)
$$

is the $a$ component of the matter energy-momentum flux. $S$ represents the spatial boundary of the volume $V$. Therefore the loss of gravitational energy is determined by the equation

$$
\frac{d E}{d t}=-\Phi_{g}^{(0)}-\Phi_{m}^{(0)}
$$

Let us consider the Lagrangian field equation (20) in the form

$$
\partial_{\nu}\left(-4 k e \Sigma^{a \lambda \nu}\right)=-k e e^{a \mu}\left(4 \Sigma^{b \nu \lambda} T_{b \nu \mu}-\delta_{\mu}^{\lambda} \Sigma^{b d c} T_{b c d}\right)-e e_{\mu}^{a} T^{\lambda \mu} .
$$

The $\lambda=0$ components of this equation may be written in terms of $\Pi^{a k}$,

$$
\partial_{k}\left(\Pi^{a k}\right)=-k e e^{a \mu}\left(4 \Sigma^{b j 0} T_{b j \mu}-\delta_{\mu}^{0} \Sigma^{b d c} T_{b c d}\right)-e e_{\mu}^{a} T^{0 \mu} .
$$

It is useful to define the quantity $\phi^{a \lambda}$,

$$
\phi^{a \lambda}=k e e^{a \mu}\left(4 \Sigma^{b c \lambda} T_{b c \mu}-\delta_{\mu}^{\lambda} \Sigma^{b d c} T_{b c d}\right) .
$$

In terms of $\phi^{a \lambda} \mathrm{Eq.} \mathrm{(27)} \mathrm{reads}$

$$
-\partial_{k} \Pi^{a k}=\phi^{a 0}+e e_{\mu}^{a} T^{0 \mu} .
$$

Integration of the equation above yields

$$
P^{a}=\int_{V} d^{3} x\left(\phi^{a 0}+e e^{a}{ }_{\mu} T^{0 \mu}\right) .
$$

Eq. (30) suggests that $P^{a}$ does indeed represent the total, gravitational and matter fields energy-momentum.

In view of Eqs. (22), (28) and (29) we have

$$
\frac{d P^{a}}{d t}=-\oint_{S} d S_{j} \phi^{a j}-\oint_{S} d S_{j}\left(e e_{\mu}^{a} T^{j \mu}\right),
$$


or

$$
\frac{d}{d t} \int_{V} d^{3} x\left(\phi^{a 0}+e e_{\mu}^{a} T^{0 \mu}\right)=-\int_{V} d^{3} x \partial_{j}\left(\phi^{a j}+e e_{\mu}^{a} T^{j \mu}\right) .
$$

We define the gravitational energy-momentum tensor $t^{\lambda \mu}$ as

$$
t^{\lambda \mu}=k\left(4 \Sigma^{b c \lambda} T_{b c}^{\mu}-g^{\lambda \mu} \sum^{b c d} T_{b c d}\right)
$$

and therefore

$$
\phi^{a \lambda}=e e^{a}{ }_{\mu} t^{\lambda \mu}
$$

In terms of $t^{\lambda \mu}$ we have

$$
\frac{d}{d t} \int_{V} d^{3} x e e^{a}{ }_{\mu}\left(t^{0 \mu}+T^{0 \mu}\right)=-\oint_{S} d S_{j}\left[e e^{a}{ }_{\mu}\left(t^{j \mu}+T^{j \mu}\right)\right] .
$$

The total space-time energy-momentum tensor $t^{\lambda \mu}+T^{\lambda \mu}$ obeys the continuity equation (35). Thus the total space-time energy-momentum is conserved for appropriate boundary conditions, if the right hand side of the equation above vanishes under integration on the whole three-dimensional space. Finally, we note that the field equations (26), which are equivalent to Einstein's equations, may be written in a simple form as

$$
\partial_{\nu}\left(e \sum^{a \lambda \nu}\right)=\frac{1}{4 k} e e^{a}{ }_{\mu}\left(t^{\lambda \mu}+T^{\lambda \mu}\right)
$$

from what follows that $\partial_{\lambda}\left[e e^{a}{ }_{\mu}\left(t^{\lambda \mu}+T^{\lambda \mu}\right)\right]=0$. Equation (30) may be alternatively given by

$$
P^{a}=\int_{V} d^{3} x e e^{a}{ }_{\mu}\left(t^{0 \mu}+T^{0 \mu}\right) .
$$

However, for practical purposes expression (21) can be handled more easily than Eq. (37).

The equation above shows that in order to arrive at values for the energymomentum $P^{a}$, the energy-momentum tensor has to be projected on a frame. Both $P^{a}$ and $t^{\lambda \mu}$ are frame dependent. We argue that it does not make sense to require quantities such as energy and momentum to be frame independent [14]. The perception of the energy of a particle at rest, say, depends on whether the observer is at rest, or is under a Lorentz boost (in flat space-time, 
taking into account the principle of relativity, the particle may be considered as undergoing a boost with respect to the observer at rest), or is accelerated. This issue has been addressed in Ref. [14], where it has been shown that for a moving observer that experiences a Lorentz boost the gravitational energy of a black hole is modified by the usual multiplicative factor $\gamma=\left(1-v^{2} / c^{2}\right)^{-1 / 2}$. The dependence of the gravitational energy with the frame also has to do with the principle of equivalence. According to the principle, an accelerated frame is locally equivalent to a rest frame, with the addition of a certain uniform gravitational field. Therefore the evaluation of the gravitational field on nearby bodies clearly depends on the state of the observer. As a consequence, a localized form of the gravitational energy (the gravitational energy contained in a finite volume of space) must also depend on the frame. We note in addition that quantities that are invariant under local (in spacetime) $\mathrm{SO}(3,1)$ symmetry are not affected by local inertial (frame) effects, and since local inertial effects are equivalent to local gravitational effects (the equivalence holds for locally uniform gravitational fields, for instance), such quantities are not expected to describe any form of localized gravitational energy.

The projection of $t^{\lambda \mu}$ on a frame, as in Eq. (37), is essential since it allows the conservation (continuity) equation (35), which follows from $\partial_{\lambda}\left[e e^{a}{ }_{\mu}\left(t^{\lambda \mu}+T^{\lambda \mu}\right)\right]=0$. We recall that in the standard formulation of general relativity the equation $\nabla_{\mu} T^{\mu \nu}=0$ for the matter energy-momentum tensor in general does not lead to conserved quantities in the space-time manifold.

The issue considered in this section has been addressed in Ref. [15]. By analyzing the field equations (20) in the vacuum space-time the authors of the latter reference arrive both at a gravitational gauge current of the YangMills type, $j_{a}{ }^{\mu}$, and at a gravitational pseudo-tensor which, according to Ref. [15], is essentially Møller's pseudo-tensor [16, 17]. The gauge current $j_{a}{ }^{\mu}$ is related to $\phi^{a \lambda}$ given by Eq. (28), which is ultimately related to the energy-momentum tensor $t^{\lambda \mu}$ according to Eq. (34). Møller's pseudo-tensor is given by Eq. (26) of Ref. [15], or, alternatively by $T_{\mu}{ }^{\nu}=\partial_{\lambda} U_{\mu}{ }^{\nu \lambda}$, where $[17]^{1}$

\footnotetext{
${ }^{1}$ As explained in Ref. [17], the latter reference presents the results of Ref. [16]
} 


$$
U_{\mu}^{\nu \lambda}=\frac{1}{8 \pi G} e\left[e^{a \nu} \nabla_{\mu} e_{a}^{\lambda}+\left(\delta_{\mu}^{\nu} e^{a \lambda}-\delta_{\mu}^{\lambda} e^{a \nu}\right) \nabla_{\sigma} e_{a}^{\sigma}\right] .
$$

In the expression above the covariant derivative is constructed out of the Christoffel symbols.

We finally remark that an expression for the energy-momentum tensor of the gravitational field that shares most of the properties of Eq. (33) has been obtained in Refs. $[19,20]$. In particular we note that the asymmetry of $t^{\lambda \mu}$ has been proved for a large class of teleparallel models in Ref. [20].

\section{The gravitational pressure}

The gravitational energy-momentum tensor yields expressions for gravitational pressures and stresses, in similarity to the energy-momentum tensor for the electromagnetic field, for instance. In this section we will show that it is possible to arrive at a consistent definition of the gravitational pressure. Restricting the following considerations to the vacuum space-time, Eq. (22) is simplified to

$$
\frac{d P^{a}}{d t}=-\Phi_{g}^{a}=-\int_{V} d^{3} x \partial_{j} \phi^{a j}=-\int_{V} d^{3} x \partial_{j}\left(e e^{a}{ }_{\mu} t^{j \mu}\right),
$$

where we have taken into account Eq. (28). By requiring the index $a$ to be a spatial index, $a=(i)=(1),(2),(3)$, we have

$$
\begin{aligned}
\frac{d P^{(i)}}{d t} & =-\int_{V} d^{3} x \partial_{j} \phi^{(i) j} \\
& =\oint_{S} d S_{j}\left(-\phi^{(i) j}\right) .
\end{aligned}
$$

In the left hand side of the equation above we have the time derivative of a momentum component, which has the character of force. Therefore the density $\left(-\phi^{(i) j}\right)$ can be understood as force per unit area, or pressure density. Specifically, $\left(-\phi^{(i) j}\right)$ can be taken as a force exerted in the $(i)$ direction on a unit area element whose normal points in the $j$ direction. Of course the right hand side of Eq. (39) can also be considered as minus the momentum flux across a surface $S$. In the case of gravitational waves for instance, as 
considered in Ref. [12], the momentum flux across a surface parallel to the wave front has the nature of a gravitational pressure.

We will make a simple application of the concept of gravitational pressure to the Schwarzschild space-time. The latter is characterized by the line element

$$
d s^{2}=-e^{2 \lambda} d t^{2}+e^{-2 \lambda} d r^{2}+r^{2} d \theta^{2}+r^{2}(\sin \theta)^{2} d \phi^{2},
$$

where $e^{2 \lambda}=1-(2 m) / r$. The set of tetrad fields adapted to an observer at rest with respect to the black hole is given by

$$
e_{a \mu}=\left(\begin{array}{cccc}
-e^{\lambda} & 0 & 0 & 0 \\
0 & e^{-\lambda} \sin \theta \cos \phi & r \cos \theta \cos \phi & -r \sin \theta \sin \phi \\
0 & e^{-\lambda} \sin \theta \sin \phi & r \cos \theta \sin \phi & -r \sin \theta \cos \phi \\
0 & e^{-\lambda} \cos \theta & -r \sin \theta & 0
\end{array}\right)
$$

It is easy to verify that in the asymptotic limit $r \rightarrow \infty$ the tetrad fields above in cartesian coordinates exhibit the asymptotic behaviour

$$
e_{\mu}^{a} \cong \delta_{\mu}^{a}+\frac{1}{2} h_{\mu}^{a}(1 / r) .
$$

A given gravitational field configuration described by the metric tensor $g_{\mu \nu}$ admits an infinity of tetrad fields, related to each other by means of a local $\mathrm{SO}(3,1)$ transformation. In order to understand how an observer is adapted to a particular set of tetrad fields, we consider its worldline in the spacetime. Let $x^{\mu}(s)$ denote the worldline $C$ of an observer, and $u^{\mu}(s)=d x^{\mu} / d s$ its velocity along $C$. We may identify the observer's velocity with the $a=(0)$ component of $e_{a}{ }^{\mu}$, where $e_{a}{ }^{\mu} e^{a}{ }_{\nu}=\delta_{\nu}^{\mu}$. Thus, $u^{\mu}(s)=e_{(0)}{ }^{\mu}$ along $C$. The acceleration of the observer is given by

$$
a^{\mu}=\frac{D u^{\mu}}{d s}=\frac{D e_{(0)}^{\mu}}{d s}=u^{\alpha} \nabla_{\alpha} e_{(0)}^{\mu} .
$$

The covariant derivative is constructed out of the Christoffel symbols. We see that $e_{a}^{\mu}$ determines the velocity and acceleration along a worldline of an observer adapted to the frame. From this perspective we conclude that a given set of tetrad fields, for which $e_{(0)}{ }^{\mu}$ describes a congruence of timelike curves, is adapted to a particular class of observers, namely, to observers determined by the velocity field $u^{\mu}=e_{(0)}^{\mu}$, endowed with acceleration $a^{\mu}$. If 
$e^{a}{ }_{\mu} \rightarrow \delta_{\mu}^{a}$ in the limit $r \rightarrow \infty$, then $e^{a}{ }_{\mu}$ is adapted to stationary observers at spacelike infinity.

Out of the set of tetrad fields given by Eq. (41) we may calculate all compoents of the torsion tensor $T_{a \mu \nu}$, of the tensor $\Sigma^{a b c}$ and consequently of $\phi^{(i) j}$. All these are long, but simple calculations.

Let us restrict considerations to a spherical surface $S$ (a surface determined by constant $r$ ). Then we have

$$
\frac{d P^{(i)}}{d t}=-\int_{S} d S_{1} \phi^{(i) 1}
$$

where we are now allowing $S$ to be an open surface. After some calculations we find

$$
\begin{aligned}
\phi^{(1) 1} & =(\sin \theta \cos \phi) 2 k \sin \theta\left[\frac{2 m}{r}\left(1-e^{\lambda}\right)-\left(1-e^{\lambda}\right)^{2}\right] \\
\phi^{(2) 1} & =(\sin \theta \sin \phi) 2 k \sin \theta\left[\frac{2 m}{r}\left(1-e^{\lambda}\right)-\left(1-e^{\lambda}\right)^{2}\right] \\
\phi^{(3) 1} & =(\cos \theta) 2 k \sin \theta\left[\frac{2 m}{r}\left(1-e^{\lambda}\right)-\left(1-e^{\lambda}\right)^{2}\right] .
\end{aligned}
$$

By defining the unit vector $\hat{\mathbf{r}}=(\sin \theta \cos \phi, \sin \theta \sin \phi, \cos \theta)$ we may express Eq. (42) for the vector quantity $\mathbf{P}=\left(P^{(1)}, P^{(2)}, P^{(3)}\right)$,

$$
\frac{d \mathbf{P}}{d t}=-\int_{S} d \theta d \phi(\sin \theta) 2 k\left[\frac{2 m}{r}\left(1-e^{\lambda}\right)-\left(1-e^{\lambda}\right)^{2}\right] \hat{\mathbf{r}} .
$$

We evaluate the integral in the equation above over a small solid angle $\Delta \Omega=$ $\sin \theta \Delta \theta \Delta \phi$ of constant radius $r$, and consider a large value of $r, r \gg m$, such that

$$
1-e^{\lambda} \approx \frac{m}{r}=\frac{M G}{c^{2} r}
$$

By making the replacements $d t \rightarrow d(c t), k=\frac{1}{16 \pi} \rightarrow \frac{c^{3}}{16 \pi G}$, we obtain

$$
\frac{d \mathbf{P}}{d t}=-\left(r^{2} \Delta \Omega\right) \frac{M^{2} G}{r^{4}} \hat{\mathbf{r}} .
$$

The quantity on the right hand side of the equation above, $-\left(M^{2} G\right) / r^{4}$, is interpreted as the gravitational pressure exerted on the area element $\left(r^{2} \Delta \Omega\right)$. 
As an interesting consequence of Eq. (45), we note that the latter equation can be rewritten as

$$
\frac{d}{d t}\left(\frac{\mathbf{P}}{M}\right)=-\frac{G M}{r^{2}} \Delta \Omega \hat{\mathbf{r}} .
$$

The left hand side of Eq. (46) may be understood as a gravitational acceleration field that acts on the solid angle $\Delta \Omega$, at a radial distance $r$.

For a different choice of tetrad fields Eqs. (45) and (46) lead to different results. It is clear that Eq. (38) transforms covariantly under a global $\mathrm{SO}(3,1)$ transformation, and so do expressions (42), (43) and (44). We can further consider a set of tetrad fields adapted to accelerated observers (the tetrad fields considered in Ref. [14], for instance), in which case expressions (45) and (46) would yield different results because of noninertial effects.

\section{Concluding remarks}

We have presented a very simple construction of the gravitational energymomentum tensor, by just considering Einstein's equations in the teleparallel description. The energy-momentum tensor yields the previously obtained expression for the gravitational energy-momentum $P^{a}$. The crucial point in the present analysis, as we pointed out earlier, is the identification of Eq. (21) as the gravitational energy-momentum, which is now understood as the total, gravitational and matter fields energy-momentum. Unlike the usual energy-momentum tensors in classical field theory [18], the energymomentum tensor considered above is unique, in the sense that it does not allow a redefinition, i.e., the addition of extra quantities, because these extra quantities would violate the field equations. An immediate issue arises regarding the definition given by Eq. (33): the energy-momentum tensor $t^{\lambda \mu}$ is not symmetric. The symmetry of energy-momentum tensors is normally related to the conservation of angular momentum [18]. The first term in Eq. (33) is asymmetric. The asymmetry of similar tensors has been addressed in Ref. [20]. It has been shown that in arbitrary teleparallel models the gravitational energy-momentum tensor cannot be purely antisymmetric, and that

for all viable models the antisymmetric part is nonvanishing. The meaning 
of the antisymmetric components of $t^{\lambda \mu}$ is not yet understood, and therefore this issue, together with the conservation of the gravitational angular momentum, deserves further investigation.

The tetrad field $e^{a}{ }_{\mu}$ in Eq. (20) has 16 components. The 6 additional components (with respect to the metric tensor $g_{\mu \nu}$ establish the reference frame of a hypothetical observer. These components characterize the rotational and translational behaviour of the frame (this issue has been discussed in section IV of Ref. [8]). For a given gravitational field configuration (a black hole space-time, for instance), the observer may be either at rest, or undergoing a Lorentz boost, or may be linearly accelerated [14].

The definition of gravitational pressure may be useful in investigations of the thermodynamics of the gravitational field. For a given gravitational field configuration we may determine the energy and pressure of a given space volume. Therefore the analysis of the thermodynamic relation $T d S=$ $d E+p d V$ may be extended from the horizon area of black holes to arbitrary volumes in space. Of course it is not straightforward to generalize the above thermodynamic relation in this sense, because the notion of gravitational entropy is so far connected with the area of the horizon of a black hole.

\section{References}

[1] F. W. Hehl, in Proceedings of the 6th School of Cosmology and Gravitation on Spin, Torsion, Rotation and Supergravity, Erice, 1979, edited by P. G. Bergmann and V. de Sabbata (Plenum, New York, 1980); F. W. Hehl, J. D. McCrea, E. W. Mielke and Y. Ne'eman, Phys. Rep. 258, 1 (1995).

[2] K. Hayashi, Phys. Lett. 69B, 441 (1977); K. Hayashi and T. Shirafuji. Phys. Rev. D19, 3524 (1979); Phys. Rev. D24, 3312 (1981).

[3] J. M. Nester, Int. J. Mod. Phys. A 4, 1755 (1989)

[4] J. W. Maluf, J. Math. Phys. 35. 335 (1994).

[5] Y. Obukhov and J. G. Pereira, Phys. Rev. D 67, 044016 (2003).

[6] L. B. Szabados, Living Rev. Relativity 7, 4 (2004). 
[7] J. W. Maluf, J. Math. Phys. 36, 4242 (1995).

[8] J. W. Maluf, J. F. da Rocha-Neto, T. M. L. Toríbio and K. H. CastelloBranco, Phys. Rev. D 65, 124001 (2002).

[9] S. V. Babak and L. P. Grishchuk, Phys. Rev. D 61, 024038 (1999).

[10] T. Regge and C. Teitelboim, Ann. Phys. (N.Y.) 88, 286 (1974).

[11] J. W. Maluf and J. F. da Rocha-Neto, Phys. Rev. D 64, 084014 (2001).

[12] J. W. Maluf, F. F. Faria and K. H. Castello-Branco, Class. Quantum Grav. 20, 4683 (2003).

[13] J. W. Maluf and F. F. Faria, Ann. Phys. (Leipzig) 13, 604 (2004).

[14] J. W. Maluf, Accelerated observers and gravitational radiation, [grqc/0412055], to appear in Gravitation and Cosmology (2005).

[15] V. C. Andrade, L. C. T. Guillen and J. G. Pereira, Phys. Rev. Lett. 84, $4533(2000)$.

[16] C. Møller, Mat. Fys. Skr. Dan. Vidensk. Selsk, 1, No. 10 (1961).

[17] C. Møller, Tetrad fields and conservation laws in general relativity, in the Proceedings of the International School of Physics "Enrico Fermi", edited by C. Møller Academic Press, London, 1962).

[18] L. D. Landau and E. M. Lifshitz, The Classical Theory of Fields (Pergamon Press, Oxford, 1980).

[19] Y. Itin, Class. Quantum Grav. 19, 173 (2002).

[20] Y. Itin, Gen. Rel. Grav. 34, 1819 (2002). 